\title{
HIGIENIZAÇÃO DAS MÃOS E A SEGURANÇA DO PACIENTE PEDIÁTRICO
}

\author{
HAND WASHING AND SAFETY OF THE PEDIATRIC PATIENT
}

\section{LAVADO DE MANOS Y SEGURIDAD DEL PACIENTE PEDIÁTRICO}

\author{
Francielle Maciel Silva* \\ Talita Padilha Porto ${ }^{* *}$ \\ Patricia KuERTEN Rocha ${ }^{* * *}$ \\ Juliana CRistina Lessmann ${ }^{* * * *}$ \\ Patricia Fernanda de A. Cabral ${ }^{* *}$ \\ Karine Larissa KNAESEl SCHNEIDER ${ }^{* * * * *}$
}

\begin{abstract}
RESUMO
Objetivo: Avaliar se a higienização das mãos realizada antes do preparo e da administração de medicamentos e fluidoterapia pelos profissionais de enfermagem segue as diretrizes estabelecidas pela Organização Mundial de Saúde (OMS) e Agência Nacional de Vigilância Sanitária (ANVISA). Material e método: Estudo quantitativo exploratório descritivo realizado de agosto a novembro de 2010, cuja coleta de dados foi implantada por meio da observação direta da higienização das mãos em uma unidade de internação pediátrica de um Hospital Universitário do Sul do Brasil, nos turnos manhã e tarde. Resultados: Constatou-se a baixa adesão à prática de higienização das mãos e estrutura inadequada, segundo as diretrizes estabelecidas pela OMS e ANVISA. Considerações finais: Há necessidade de se reestruturar o espaço físico e fornecer condições favoráveis para realização das técnicas de higienização das mãos pelos profissionais. Como também promover capacitação e educação continuada, garantindo, assim, a segurança do paciente pediátrico por meio da higienização das mãos.
\end{abstract}

Palavras chave: Segurança do paciente, medidas de segurança, cuidados de enfermagem, lavagem de mãos, infecção hospitalar.

\begin{abstract}
Objective: To evaluate whether the hygienisation of hands held before the preparation and administration of medication and fluids by nursing professionals follows the guidelines established by the World Health Organization (WHO) and the National Health Surveillance Agency (ANVISA). Methods: This is an exploratory descriptive quantitative study conducted between August and November 2010, whose data collection was implemented through direct observation of hand washing procedure in a pediatric unit at a University Hospital in southem Brazil, during morning and afternoon shifts. Results: Low adherence to the practice of hand hygiene

\footnotetext{
*Enfermeira. Membro do Grupo de Pesquisa GAPEFAM. Email: escoteiracia@hotmail.com

${ }^{* *}$ Enfermeira. Membro do Grupo de Pesquisa GAPEFAM. Email: talitap2@hotmail.com

*** Enfermeira. Docente da Graduação e do Programa de Pós-graduação do Departamento de Enfermagem da UFSC. Membro do Grupo de Pesquisa Clínica, Tecnologias e Informática em Saúde e Enfermagem - GIATE e pesquisadora colaboradora do GEPESCA. Email: pkrochaucip@gmail.com

${ }^{* * * *}$ Enfermeira. Membro do Núcleo de Estudos e Assistência em Enfermagem e Saúde à Pessoas em Condição Crônica NUCRON. Email: julianalessmann@gmail.com

***** Enfermeira. Membro do Grupo de Pesquisa Clínica, Tecnologias e Informática em Saúde e Enfermagem - GIATE. Membro do GEPESCA. Email: patifac@yahoo.com.br

****** Enfermeira da Unidade de Internação Pediátrica - HU/UFSC. Membro do Núcleo de Pesquisa e Estudos sobre Enfermagem, Quotidiano, Imaginário e Saúde de Santa Catarina - NUPEQUIS. Email: kalarissa@ibest.com.br
} 
and inadequate structure, according to the guidelines set by WHO and ANVISA, was noted. Conclusion: There is a need to restructure the physical space and provide favorable conditions for carrying out the techniques of hand washing by health professionals. As well, promote continuing education and training with the team, ensuring pediatric patients safety through hand hygiene.

Key words: Patient safety, security measures, nursing care, hand washing, cross infection.

\section{RESUMEN}

Objetivo: Evaluar si la higiene de las manos realizada antes de la preparación y administración de medicamentos y líquidos por el personal de enfermería sigue las directrices establecidas por la Organización Mundial de la Salud (OMS) y la Agencia Nacional de Vigilancia Sanitaria (ANVISA). Material y método: Es un estudio cuantitativo, exploratorio, realizado de agosto a noviembre de 2010, cuya recolección de datos se hizo mediante la observación aleatoria en los turnos de mañana y tarde. Resultados: Se encontró poca adherencia a la práctica de la higiene de las manos y estructura inadecuada, según las directrices establecidas por la OMS/ANVISA. Conclusión: Hay necesidad de restructurar el espacio físico, brindar condiciones favorables para la realización de las técnicas de lavado de las manos por los profesionales, promover la educación continua y capacitación con el equipo para garantizar la seguridad de los pacientes pediátricos mediante la higiene de las manos.

Palabras clave: Seguridad del paciente, medidas de seguridad, atención de enfermería, lavado de manos, infección hospitalaria.

Fecha recepción: 03/07/12 Fecha aceptación: 06/06/13

\section{INTRODUÇÃO}

Desde a antiguidade têm-se que a higienização das mãos é considerada um ato de limpeza e purificação, porém, somente após a descoberta dos microrganismos nas superfícies corporais, é que a preocupação da higienização das mãos foi tida como forma de combate à transmissão de doenças $(1,2)$.

Como as mãos são as principais ferramentas de trabalho dos profissionais que atuam nos serviços de saúde, a segurança do paciente depende diretamente da realização frequente e correta da prática de higienização das mãos (3).

Embora o objetivo do cuidado seja promover saúde e recuperação do paciente, esse está sujeito a riscos decorrentes da assistência prestada. Por esse motivo, busca-se a qualidade e a segurança na prestação de serviços. Assim, a Organização Mundial da Saúde criou em 2004 a Aliança Mundial pela Segurança do Paciente, instituindo uma agenda com 12 áreas de atuação. Sendo a primeira intitulada como "Desafios Globais para Segurança do Paciente", na qual uma das diretrizes priorizadas é o "Cuidado Limpo é Cuidado Seguro" $(4,5)$.

No ano de 2007, o Brasil se juntou a Aliança Mundial para Segurança do Paciente, e declarou seu compromisso na luta contra as infecções relacionadas à assistência à saúde, iniciativa proposta pelo programa "Desafio Global de Segurança do Paciente" que tem como área de trabalho a higienização das mãos, assim, as diretrizes estabelecidas pelo desafio "Uma Assistência Limpa é uma Assistência mais Segura" - Clean care is safer care, sofre adaptação e tradução para ser utilizada no Brasil, e começa a ser implementada em diversas instituições nacionais (6).

A infecção associada ao cuidado de saúde é vista como uma doença grave, com grande impacto econômico e social para os pacientes e os sistemas de saúde em todo o mundo. Em Trinidad e Tobago, na América Central, os gastos com infecções representam 5\% do 
orçamento anual de um hospital. Na Tailândia, no Sudeste Asiático, o gasto de alguns hospitais chega a $10 \%$ de seu orçamento anual para coibir as infecções (7).

$\mathrm{O}$ impacto é maior entre os pacientes mais vulneráveis. Há registro que em países em desenvolvimento, todos os dias, 4384 crianças morrem por decorrência de infecções relacionadas à assistência a saúde (8).

Todavia, historicamente há a constatação do valor da higienização das mãos na prevenção das doenças, porém, os profissionais de saúde continuam ignorando a importância desta prática tão simples e não compreendem os mecanismos básicos da dinâmica de transmissão de micro-organismos que podem provocar o desenvolvimento de infecções (2).

Vê-se que publicações da Organização Mundial da Saúde demonstram que centenas de milhões de pacientes em todo o mundo são acometidos por infecções associadas ao cuidado de saúde, e que por meio de estratégias simples, como a higienização das mãos, pode-se reduzir consideravelmente esse número $(9,10)$.

É importante salientar que o termo "lavagem das mãos" foi substituído por "higienização das mãos", que compreende a higienização simples, a higienização anti-séptica, a fricção anti-séptica e a anti-sepsia cirúrgica das mãos, ampliando assim, a abrangência desse termo (11).

Nesse sentido, é indicado que esse ato seja realizado em cinco momentos: 1 ) antes de contato com o paciente; 2) antes da realização de procedimento asséptico; 3 ) após risco de exposição a fluidos corporais; 4) após contato com o paciente; e 5) após contato com as áreas próximas ao paciente (12).

Estudo multicêntrico realizado durante três anos de observação em quatro hospitais, demonstrou que aplicando-se intervenções multimodais, foi possível aumentar os índices de higienização das mãos e uso de luvas de maneira adequada, e por consequência, obter redução significativa na incidência de bactérias multirresistentes no ambiente hospitalar (13).

Demonstra-se que a adesão a práticas seguras como a higienização das mãos simples e a friç̧ão anti-séptica diminui os riscos aos pacientes. Em se tratando de pediatria, acrescenta-se o fator de que as crianças apresentam o sistema imunológico ainda em desenvolvimento, sendo assim mais suscetíveis às infecções (14).

Diante do exposto, a higienização das mãos é uma medida simples que deve ser amplamente utilizada pelos profissionais da saúde, diminuindo os riscos ao paciente e os custos globais relacionados à assistência à saúde, resultando em um maior nível de segurança ao paciente, especialmente, o pediátrico.

Sendo assim, este estudo teve como objetivos avaliar se a higienização das mãos realizada antes do preparo e da administração de medicamentos (por via intravenosa, intramuscular, subcutânea e oral) ou fluidoterapia pelos profissionais de enfermagem segue o preconizado pela Organização Mundial da Saúde (15) e pela Agência Nacional de Vigilância Sanitária (16), e também, avaliar as características físicas e estruturais da unidade.

\section{MATERIAL E MÉTODO}

Trata-se de um estudo quantitativo do tipo exploratório descritivo, realizado em uma unidade de internação pediátrica de um Hospital Universitário do Sul do Brasil, no período de agosto à novembro de 2010, nos turnos matutino e vespertino.

A coleta de dados foi implementada por observação direta do procedimento de higienização das mãos, propiciada pelo instrumento elaborado. Esse método foi escolhido por ser o único que resulta no número exato de vezes em que o profissional realiza a higienização das mãos, como também proporciona a análise do procedimento (17). 
Para a coleta de dados, foi elaborado um instrumento de observação fundamentado no Guide to Implementation - A Guide to the implementation of the WHO Multimodal Hand Hygiene Improvement Strategy (15); e no Guia para Implementação da estratégia multimodal da OMS para a melhoria da higienização das mãos - ANVISA(16). Este instrumento foi dividido em 3 áreas de observação, a saber: estrutura da unidade; instrução para lavagem correta das mãos; e técnica de higienização das mãos realizada.

A validação do instrumento foi realizada por meio de pré-teste em 10 observações, que mostrou as congruências e divergências deste. Após os ajustes necessários, procedeu-se a coleta de dados. E para os dados de caracterização da população do estudo, utilizou-se a entrevista.

Como critérios de inclusão: ser profissional da equipe de enfermagem da unidade; estar no exercício de suas funções no período da coleta de dados e aceitar participar do estudo. Como critérios de exclusão: declínio do termo de consentimento.

O projeto foi apresentado aos profissionais, em seus objetivos e métodos, porém, sem revelar o que seria observado e analisado pelas pesquisadoras. Após essa breve apresentação, foi realizado o convite para os profissionais e em seguida, a entrega do Termo de Consentimento Livre e Esclarecido para assinatura.

Assim, participaram 13 profissionais da equipe de enfermagem que realizavam o preparo e administração de medicamentos e fluidoterapia no período em que duas pesquisadoras encontravam-se na unidade para a coleta de dados. A observação se deu de maneira direta e não participante, sendo que as pesquisadoras se posicionavam na sala de preparo de medicação, de maneira a não interferir no procedimento de higienização das mãos. O tempo médio de observação foi de 10 horas por dia, em cerca de 15 dias por mês, resultando em cerca de 600 horas de observação e 209 oportunidades de higie- nização das mãos.

Os dados foram tabulados em planilha utilizando a ferramenta Microsoft Excel ${ }^{\circledR}$ de 2003, sendo realizada estatística descritiva simples, como a frequência, média, e desvio padrão por meio do SESTATNET ${ }^{\circledR}$ da Universidade Federal de Santa Catarina.

O estudo foi aprovado pelo Comitê de Ética e Pesquisa da Instituição, conforme parecer 853/10.

\section{RESULTADOS}

Foram realizadas 209 observações de oportunidade de higienização das mãos no processo de preparo e administração de medicamentos e fluidoterapia, sendo que em 87 $(42,63 \%)$ observações foi realizada a higienização das mãos, dentre estas, em 66 (75,86\%) foi realizada a higienização das mãos com água e sabão, em $8(9,20 \%)$ efetuada a fricção antisséptica das mãos, e em 13 (14,94\%) foram combinadas as duas técnicas.

Quanto a caracterização dos participantes do estudo, a média de idade foi de 39.9 anos, com desvio padrão (DP) de 9.1 anos. Quanto ao tempo de formação, obteve-se média de 16.3 anos com DP de 9.4 anos, e a média de tempo de trabalho foi de 14.4 anos, com DP de 6.8 anos. Dos 13 profissionais de enfermagem observados, 12 (92,31\%) eram técnicos de enfermagem e $1(7,69 \%)$ era enfermeira. Houve predominância do sexo feminino com total de 12 (92,31\%) mulheres.

Quanto à infra-estrutura, a unidade possui 16 pias com torneiras manuais de água potável e limpa, sendo que destas, 6 são para o uso exclusivo dos profissionais e se localizam no expurgo, sala de medicação, sala de lanche, sala de procedimentos e sala de nutrição; e 10 pias encontram-se disponíveis para o uso das crianças, acompanhantes e profissionais, estando dispostas de diferentes maneiras, ou seja, estas atendem onze quartos e encontram-se dentro de alguns deles, 
na ante-sala de outros e na brinquedoteca. Os porta papéis toalhas são fabricados em material plástico, de fácil higienização, fixados ao lado de cada pia, em altura que respingos de água não os atingem.

Em relação ao dispensadores de álcool gel, encontramos um total de 12 , sendo que os mesmos encontram-se fixados pela unidade, como: sala de observação, brinquedoteca, ante-sala dos quartos, corredor central e no interior de alguns quartos.

Foi identificado que não há cartazes de promoção de higienização das mãos expostos na unidade. Além disso, os funcionários observados não receberam instrução específica sobre higienização das mãos nos últimos dois anos, exceto uma funcionária que cursa graduação em enfermagem, que por meio desta teve a oportunidade de receber há menos de um ano, uma capacitação dentro dos parâmetros do referido curso, sendo que as autoras não foram informadas acerca de que maneira o assunto foi abordado.

Quanto às lixeiras, todos os quartos e ambientes da unidade possuem esta com pedal, exceto a sala de preparo da medicação. Nesta sala, a lixeira diferencia-se das outras, pois possui local adequado para descartar lixo em forma de papel, plástico, vidro e lixo contaminado, porém as tampas são de madeira e manuais.

Quanto ao procedimento de higienização das mãos com água e sabão, combinado ou não ao uso do álcool em gel, foi observado que dos 79 procedimentos, $77(97,47 \%)$ foram realizados por técnicos de enfermagem e $2(3,63 \%)$ por enfermeira. Sendo que os resultados apontam que estes foram realizados no momento do preparo e/ou na administração do medicamento (Tabela 1).

Tabela 1. Realização da higienização das mãos com água e sabão antes do preparo e da administração de medicamento. Florianópolis, 2010.

\begin{tabular}{lcc}
\hline Oportunidade para Higienização das mãos & N (209) & \% \\
\hline Higienização apenas antes do preparo & 55 & 26,33 \\
Higienização apenas antes da administração & 9 & 4,32 \\
Higienização nos dois momentos & 2 & 0,91 \\
\hline Não realizada & 143 & 68,44 \\
\hline Total & 209 & 100 \\
\hline
\end{tabular}

Quanto a caracterização dos tipos de vias de administração dos medicamentos observados, $1(0,48 \%)$ foi por via intramuscular, $90(43,06 \%)$ por via intravenosa, 84 $(40,19 \%)$ por via oral, $2(0,96 \%)$ por via subcutânea, e 32 (15,31\%) foram fluidoterapia (Tabela 2 ).

Ao compararmos a via de administração com a higienização das mãos antes do preparo do medicamento (Tabela 2), verificamos que há uma paridade no total de administração de medicamentos entre as via oral e intravenosa, porém a higienização das mãos ocorreu em $38(18,18 \%)$ casos na via intravenosa e em $19(9,09 \%)$ na via oral, por meio da higienização simples (com água e sabão). Assim, verificou-se uma maior preocupação dos funcionários em relação à via intravenosa, ao compararmos com a via oral (Tabela 2). 
Tabela 2. Higienização das mãos segundo via de administração. Florianópolis, 2010.

\begin{tabular}{|c|c|c|c|c|c|c|c|c|c|c|c|c|}
\hline \multirow{3}{*}{ Higienização das mãos } & \multicolumn{12}{|c|}{ Via de administração de medicação } \\
\hline & \multicolumn{2}{|c|}{$\operatorname{IM}(01)$} & \multicolumn{2}{|c|}{ IV (90) } & \multicolumn{2}{|c|}{$\mathrm{VO}(84)$} & \multicolumn{2}{|c|}{ SC (02) } & \multicolumn{2}{|c|}{ Fluido $^{\star}(32)$} & \multicolumn{2}{|c|}{ Total (209) } \\
\hline & $n$ & $\%$ & $n$ & $\%$ & $n$ & $\%$ & $n$ & $\%$ & $n$ & $\%$ & $n$ & $\%$ \\
\hline \multicolumn{13}{|l|}{ Antes do preparo } \\
\hline Sim & 0 & 0 & 38 & 18,18 & 19 & 9,09 & 1 & 0,47 & 10 & 4,78 & 68 & 32,54 \\
\hline Não & 1 & 0,48 & 52 & 24,88 & 65 & 31,10 & 1 & 0,47 & 22 & 10,53 & 141 & 67,46 \\
\hline \multicolumn{13}{|l|}{ Antes da administração } \\
\hline $\operatorname{Sim}$ & 0 & 0 & 11 & 5,26 & 4 & 1,91 & 0 & 0 & 0 & 0 & 15 & 7,18 \\
\hline Não & 1 & 0,48 & 79 & 37,80 & 80 & 38,28 & 2 & 0,96 & 32 & 15,31 & 194 & 92,82 \\
\hline
\end{tabular}

* Fluidoterapia

Com relação à higienização antes da administração pela via intravenosa, foi observada a sua ocorrência em 11 observações (5,26\%). Enquanto que em 79 (37,8\%) das observações quanto a administração dos medicamentos pela via intravenosa não existiu qualquer higiene prévia (Tabela 2).

Quanto a técnica da higienização simples das mãos, das 209 oportunidades, verificou-se que o procedimento foi realizado isoladamente, em 66 (31,58\%) vezes, destes, $55(83,33 \%)$ antes do preparo da medicação, $9(13,64 \%)$ antes da administração, e 2 $(3,03 \%)$ nos dois momentos, ou seja, antes do preparo e da administração do medicamento.

Ainda, em 17 (25,762\%) das vezes em que as mãos foram higienizadas com água e sabão foram retiradas as jóias e relógios; as unhas estavam curtas em 55 (83,33\%); em $30(45,45 \%)$ vezes havia presença de esmalte que em $18(60 \%)$ observações não estava íntegro, e em 12 (40\%) eram de cor escura.

$\mathrm{Na}$ realização da técnica de higienização simples das mãos, viu-se que dos 11 passos preconizados pela ANVISA (2007), em 4 destes, uma média de 58 (87,89\%) higienizações não foram realizadas conforme a técnica determinada (Tabela 3). Além dos passos preconizados, ainda foi observado se o fechamento das torneiras manuais ocorriam com papel toalha, e verificado que em 55 $(83,33 \%)$ vezes o procedimento foi realizado adequadamente.

Tabela 3. Técnica de higienização simples das mãos, Florianópolis, 2010.

\section{Técnica de higienização simples das mãos}

Abre a torneira e molha as mãos, evitando encostar-se a pia

Sim

Não

Aplica na palma da mão quantidade suficiente de sabonete líquido para cobrir todas as superfícies das mãos seguindo a quantidade recomendada pelo fabricante

Sim

Não

$\mathrm{N}(79) \quad \%$

$73 \quad 92,41 \%$

$6 \quad 7,59 \%$ 
Continuação Tabela 3.

Ensaboa as palmas das mãos e esfrega friccionando-as entre si

Sim

$98,73 \%$

Não

Esfrega a palma da mão direita contra o dorso da mão esquerda entrelaçando os dedos e vice-versa

Sim

Não

Entrelaça os dedos e fricciona os espaços interdigitais

Sim

Não

Esfrega o dorso dos dedos de uma mão com a palma da mão oposta, segurando os dedos, com movimento de vai-e-vem e vice-versa

\section{Sim}

Não

Esfrega o polegar direito, com o auxílio da palma da mão esquerda, utilizando-se de movimentos circular e vice-versa

\section{Sim}

Não

Fricciona as polpas digitais e unhas da mão direita contra a palma da mão direita fazendo movimento circular e vice-versa

\section{Sim}

Não

Esfrega os punhos com movimentos circulares

\section{Sim}

Não

Realiza o enxágue satisfatório das mãos com água, retirando os resíduos de sabão.

Evite o contato direto das mãos ensaboadas com a torneira.

\begin{tabular}{lrr} 
Sim & 78 & $98,73 \%$ \\
Não & 1 & $1,27 \%$ \\
Seca as mãos com papel-toalha descartável, iniciando pelas mãos e seguindo pelos & \\
punhos. Desprezar o papel-toalha na lixeira para resíduos comuns & \\
Sim & 79 & $100 \%$ \\
Não & 0 & $0 \%$ \\
\hline
\end{tabular}

A fricção anti-séptica das mãos ocorreu, isoladamente, em $8(3,82 \%)$ observações, combinada à higienização simples das mãos em 13 (6,23\%) e em 188 (89,95\%) das observações não foi utilizado solução alcoólica anteriormente ao contato com o paciente.

Ao avaliar o tempo de higienização simples das mãos e de fricção alcoólica, antes do preparo e da administração do medicamento ou fluidoterapia, tem-se que a higienização durou até 10 segundos em 13 (14,94\%) observações, de 11 à 20 segundos em 58 $(66,67 \%)$ dos casos, de 21 à 30 segundos em $15(17,24 \%)$ observações, e mais de 30 segundos em 1 (1,15\%) observação. 


\section{DISCUSSÃO E CONCLUSÃO}

A Organização Mundial da Saúde em 2007 constatou que entre 5 e $10 \%$ dos pacientes admitidos em hospitais adquirem uma ou mais infecções, e a higienização das mãos é a medida primária para a redução dessa taxa quando relacionada à assistência de saúde (18). A contaminação das mãos da equipe de saúde pode acontecer por meio do contato direto com o paciente, ou por meio indireto, por meio do uso de equipamentos durante a assistência ou ao tocar nos mobiliários (19, 20). Os índices baixos de higienização das mãos encontrados nesta pesquisa, nos remeteu a uma preocupação com o número de casos de infecção que podem ser adquiridos devido a esta prática inadequada.

Ainda verificou-se que a técnica de higienização simples das mãos foi mais utilizada que a friç̧ão antisséptica, dessa forma, podemos constatar que ainda não está incutido na cultura destes profissionais o uso do antisséptico como um agente que substitui a higienização simples das mãos em momentos da assistência.

Deve-se observar que a utilização do álcool-gel apresenta vantagens que contribuem para um cuidado mais seguro, como a economia no tempo de realização da prática e a não agressividade da pele do profissional, pois a solução alcoólica não retira a flora microbiana da pele $(11,21)$.

Quanto aos aspectos ambientais, a ANVISA aponta que os lavatórios ou pias devem possuir torneiras que dispensem o uso das mãos para o fechamento da água (22). Como podemos constatar nos resultados, todas as torneiras utilizadas na unidade são manuais.

A proporção definida pela RDC $\mathrm{n}^{0} 50$ de 2002 consiste em um lavatório externo que pode servir a no máximo 4 quartos ou duas enfermarias. Além disso, cada quarto ou enfermaria deve possuir um banheiro exclusivo, além de um lavatório/pia para uso da equipe de cuidado em uma área anterior a entrada do quarto/enfermaria ou mesmo no interior desses, fora do banheiro. Essas determinações não são totalmente atendidas no ambiente de estudo, assim, pode dificultar a adesão dos profissionais da equipe de enfermagem para com a higienização simples das mãos antes dos procedimentos e contato com os pacientes (22).

Verificamos que o procedimento de higienização simples das mãos foi mais observado antes do preparo do medicamento que antes de sua administração, o que podemos atribuir ao fácil acesso à pia constante na sala de preparo de medicação.

Nesse sentido, a falta de adesão à higienização das mãos e as condições não adequadas do ambiente estudado interferem diretamente na segurança do paciente pediátrico, podendo ocasionar infecções e eventos adversos à saúde dessas crianças (23).

Outro aspecto imprescindível, é que junto aos lavatórios e as pias exista recipiente para o recolhimento do material utilizado na secagem das mãos, é necessário que tal recipiente seja de limpeza fácil, não precisando ter tampa. Caso a opção seja por conservá-lo fechado, o mesmo terá que possuir tampa articulada com sistema de abertura sem o uso das mãos (24). No entanto, na sala de preparo de medicação, a lixeira utilizada para descarte desses materiais contém tampas que necessitam do uso das mãos para o manuseio.

Apesar de pesquisas demonstrarem que as mãos exercem considerável relação com a transmissão de micro-organismos infecciosos e que os procedimentos de higienização das mãos implicam na redução das taxas de infecção, muitos profissionais e administradores hospitalares continuam agindo de forma passiva diante dessa dificuldade, entretanto outros poucos criam formas originais e inovadoras para chamar a atenção dos profissionais em campanhas educativas de higienização das mãos (25-28).

Verificamos que mesmo a higienização das mãos sendo uma premissa à assistência, 
muitas vezes ela não é realizada adequadamente. Isto nos leva a pensar em estratégias para transformar esta realidade e elevar os índices de segurança do paciente pediátrico, como exemplos podemos citar: fornecer condições adequadas aos funcionários que prestam o cuidado diário aos pacientes, de modo a alterar significativamente o quadro encontrado naquela realidade; fomentar um maior uso do álcool-gel pelos funcionários como forma eficaz de higienizar as mãos; promover capacitações e qualificações aos profissionais para que possam realizar a técnica de higienização das mãos de maneira correta.

Assim, existe a necessidade da implantação de uma estrutura de trabalho adequada aos profissionais atuantes não somente no caso em estudo, mas em muitas outras unidades que passam por situações semelhantes. Fornecer locais equipados com pias e dispensadores de álcool-gel de fácil acesso, e a adoção de frascos de bolso da solução antisséptica, possibilita a adesão dos profissionais a preocuparem-se com a segurança do paciente e consequentemente com a própria segurança.

Outra questão fundamental é a educação permanente e a atualização destes profissionais, prepará-los com capacitações que expliquem a importância de realizar a higienização das mãos, as consequências da não realização do procedimento, o que significa cultura e segurança do paciente, entre outros temas que os incentivem a prestar um cuidado mais seguro, contribuirá para atenuar os altos índices de infecção e contaminação, garantindo, desse modo, uma assistência de qualidade ao paciente pediátrico.

Tendo em vista os achados aqui relatados, é de grande importância que os profissionais da saúde se conscientizem que através de uma técnica simples, como a higienização das mãos, é possível elevar o cuidado prestado ao paciente pediátrico a um cuidado seguro, minimizando os danos provenientes da assistência em saúde.

\section{REFERÊNCIAS}

1. Andrade GM. Infecção hospitalar: mitos e verdades, velhos hábitos, novas atitudes. Brasília méd. 2002; 39(1/4): 57-9.

2. Mendonça AP, Fernandes MSC, Azevedo JMR, Silveira WCR, Souza ACS. Lavagem das mãos: adesão dos profissionais de saúde em uma unidade de terapia intensiva neonatal. Acta scientiarum. Health sciences. 2003; 25(2): 147-53.

3. Brasil. Agência Nacional de Vigilância Sanitária. Segurança do paciente: higienização das mãos. Brasília, DF: Ministério da Saúde; 2008.

4. Gomes AQF. Iniciativas Globais para Segurança do Paciente. Rio de Janeiro: Fundação Oswaldo Cruz, PROQUALIS; 2010.

5. World Health Organization. World Alliance for Patient Safety: Foward Programme 2008-2009 [Internet]. Geneva: WHO; 2008 [accesado 08 junio 2012]. 64 p. Disponível em: http://www.who. int/patientsafety/information_centre/ reports/Alliance_Forward_Programme_2008.pdf

6. Brasil. Agência Nacional de Vigilância Sanitária. Um guia para implantação da estratégia multimodal da OMS para a melhoria da higienização das mãos; 2006/2007.

7. Organização Mundial Da Saúde. Diretrizes da OMS sobre higienização das mãos na assistência à saúde (versão preliminar avançada): resumo. Geneva: WHO; 2005. $34 \mathrm{p}$.

8. World Health Organization Collaborating Centre for Patient Safety Solutions. Improved Hand Hygiene to Prevent Health Care-Associated Infections. Patient Safety Solutions [Internet]. 2007 [acessado 08 junio 2012]; 1(9): 1-4. Disponível em: http://www.who.int/patientsafety/ solutions/patientsafety/PS-Solution9. pdf. 
9. Allegranzi B, Nejad SB, Combescure C, Graafmans W, Attar H, Donaldson L et al. Burden of endemic health-care-associated infection in developing countries: systematic review and meta-analysis. Lancet. 2011; 377(9761): 228-41.

10. World Health Organization. Report on the burden of endemic health care-associated infection worldwide: Clean Care is Safer Care. Geneva: WHO; 2011.34p.

11. Centers for Disease Control and Prevention. Guideline for Hand Hygiene in Health-Care Settings: Recommendations of the Healthcare Infection Control Practices Advisory Committee and the HICPAC/SHEA/APIC/IDSA Hand Hygiene Task Force. Atlanta: MMWR; 2002. (Publicación científica Vol. 51 / No. RR-16).

12. World Health Organization, Patient Safety a World Alliance for Safer Health Care. Conceptual Framework for the International Classification for Patient Safety, version 1.1: Final Technical Report [Internet]. Geneve: WHO; 2009 [accesado 28 janeiro 2012]. 154 p. Encontrado em: http://www.who.int/patientsafety/taxonomy/icps_full_report.pdf

13. Trick WE, Vernon MO, Welbel SF, Demarais P, Hayden MK, Weinstein RA. Multicenter intervention program to increase adherence to hand hygiene recommendations and glove use and to reduce the incidence of antimicrobial resistance. Infect Control Hosp Epidemiol. 2007; 28(1): 42-9.

14. Ministério da Saúde, Agência Nacional de Vigilância Sanitária. Pediatria: Prevenção e controle de infecção hospitalar. Brasília: Editora Anvisa; 2005. 116 p.

15. World Health Organization, Patient Safety a World Alliance for Safer Health Care. Guide to Implementation: A guide to the Implementation of the WHO Multimodal Hand Hygiene Improvement Strategy [Internet]. Geneva: WHO; 2009 [acessado 09 dezembro 2011]. 48 p. Encontrado em: http://www.who.int/gpsc/5may/Gui-
de_to_Implementation.pdf

16. Agência Nacional de Vigilância Sanitária. Higienização das mãos em serviços de saúde. Brasília: Anvisa; 2007. 52p.

17. Bolon M. Handy hygiene. Infect Dis Clin North Am. 2011; 25(1): 21-43.

18. World Health Organization. World Alliance for Patient Safety: Foward Programme 2006-2007. Geneva: WHO; 2006. 64p.

19. Chakrabarti A, Singh K, Narang A, Singhi S, Batra R, Rao KL et al. Outbreak of Pcihia anomala infection in the pediatric service of a tertiary-care Center in Northern India. J Clin Microbiol. 2001; 39(5): 1702-6.

20. Levin AS, Costa SF, Mussi NS, Basso M, Sinto SI, Machado C, et al. Candida parapsilosis fungemia associated with implantable and semi-implanted central venous catheters and the hands of healthcare workers. Diagn Microbiol Infect Dis. 1998; 30(4): 243-9.

21. World Health Organization, World Alliance for Patient Safety. WHO Guidelines on Hand Hygiene in Health Care (Advanced Draft) - Global Patient Safety Challenge 2005-2006: Clean care is safer care. Geneva: WHO Press; 2006. 209 p.

22. Dispõe sobre Normas e Projetos Físicos de Estabelecimentos Assistenciais de Saúde de 2002, Resolução RDC n ${ }^{0}$ 50, de 2002. Diário Oficial da União, Brasília: DF; 2002.

23. Dispõe sobre o Regulamento Técnico para o gerenciamento de resíduos de serviços de saúde de 2004, RDC n 306 (07 de dezembro de 2004). Diário Oficial da União, Brasília: DF; 2002.

24. Rosenthal T, Erbeznik M, Padilla T, Zaroda T, Nguyen D, Rodriguez M. Observation and Measurement of Hand Hygiene and Patient Identification Improve Compliance With Patient Safety Practices. Acad Med. 2009; 84(12): 1705-12.

25. Pittet D, Hugonnet S, Harbarth S, Mourouga $\mathrm{P}$, Sauvan V, Touveneau $\mathrm{S}$ et al. 
Effectiveness of a hospital wide program to improve compliance with hand hygiene. Lancet. 2000; 356(9238): 1307-12.

26. Voss A, Widmer AF. No time for handwashing? Handwashing versus alcoholicrub: can we afford $100 \%$ compliance? Infect Control Hosp Epidemiol. 1997; 18(3): 205-8.
27. Pittet D. Segurança do Paciente - Aliança Mundial da OMS. Anais do 1. Encontro Nacional de Vigilância Sanitária; 2009.

28. Cruz EDA, Pimenta FC, Palos MAP, Silva SRM, Gir E. Higienização de mãos: 20 anos de divergências entre a prática e o idealizado. Cienc. enferm. 2009; XV(1): 33-8. 